AperTO - Archivio Istituzionale Open Access dell'Università di Torino

Effects of intranasal oxytocin on long-term memory in healthy humans: A systematic review

This is a pre print version of the following article:

Original Citation:

Availability:

This version is available http://hdl.handle.net/2318/1609943

since 2017-03-15T12:04:55Z

Published version:

DOI:10.1002/ddr.21343

Terms of use:

Open Access

Anyone can freely access the full text of works made available as "Open Access". Works made available under a Creative Commons license can be used according to the terms and conditions of said license. Use of all other works requires consent of the right holder (author or publisher) if not exempted from copyright protection by the applicable law. 


\title{
Effects of Intranasal Oxytocin on Long-Term Memory in Healthy Humans: a Systematic Review
}

\author{
Michela Brambilla ${ }^{1}$, Rosa Manenti ${ }^{1}$, Giovanni de Girolamo ${ }^{2}$, Mauro Adenzato ${ }^{3,4,5}$, Luisella \\ Bocchio-Chiavetto $^{6,7}$, Maria Cotelli ${ }^{1}$
}

${ }^{1}$ Neuropsychology Unit, IRCCS Saint John of God Clinical Research Centre, Brescia, Italy.

${ }^{2}$ Psychiatric Epidemiology and Evaluation Unit, IRCCS Saint John of God Clinical Research

Centre, Brescia, Italy.

${ }^{3}$ Department of Psychology, University of Turin, Turin, Italy

${ }^{4}$ Center for Cognitive Science, University of Turin, Turin, Italy

${ }^{5}$ Neuroscience Institute of Turin, Turin, Italy

${ }^{6}$ Genetics Unit, IRCCS Saint John of God Clinical Research Centre, Brescia, Italy;

${ }^{7}$ Faculty of Psychology, eCampus University, Novedrate (Como), Italy

Corresponding Author:

Maria Cotelli, $\mathrm{PhD}$

IRCCS Centro San Giovanni di Dio, Fatebenefratelli; Via Pilastroni, 425125 Brescia, Italy Ph: 0039-0303501593; Fax: 0039-0303533513; Email: mcotelli@fatebenefratelli.eu

Authors have no conflict of interest.

Running title: Oxytocin and Memory

Two Tables, one Figure 


\begin{abstract}
Neuropeptide oxytocin is implicated in complex emotional and social behavior and it appears to play an important role in learning and memory. Animal studies have shown that the effects of exogenous oxytocin on memory vary according to the timing of administration, context, gender and dose and it may improve the memory of social, but not non-social stimuli. Oxytocin is intricately involved in a broad array of neuropsychiatric functions and may therefore be a pharmacological target for several psychiatric disorders. This review summarizes the potential effects of oxytocin on long-term memory processes in healthy humans. The effects of intranasal oxytocin on human memory is controversial and the studies included in this review have applied a variety of learning paradigms, in turn, producing variable outcomes. Specifically, data on the long-term memory of non-emotional stimuli found no effect or even worsening in memory, while studies using emotional stimuli showed an improvement of long-term memory performance. In conclusion, this review identified a link between long-term memory performance and exogenous intranasal oxytocin in humans, although these results still warrant further confirmation in large, multicenter randomized controlled trials.
\end{abstract}

Keywords: learning, endogenous oxytocin, encoding, recognition, recall 


\section{INTRODUCTION}

Long-term memory refers to the ability to encode, store, retain and subsequently recall information and past experiences [Gabrieli 1998]. In particular, recognition of and discrimination between conspecifics are essential behavioral components in the repertoire of memory for social stimuli in animals such as mammals, to enable appropriate social interactions. Findings from animal and human studies point unequivocally to the involvement of oxytocin in the development and maintenance of attachment and affiliative behavior [Lim and Young 2006]. Beside its essential role for mammalian parturition and lactation, oxytocin is implicated in complex emotional and social behavior and appears to play a role in both learning and memory [Gulpinar and Yegen 2004; Heinrichs et al. 2004]. Memory impairment is a particularly relevant issue for pharmacological research, since it may be associated with many psychiatric and neurological disorders [Hill et al. 2013; Koen and Yonelinas 2014; Petersen et al. 2014; Rock et al. 2014].

Oxytocin is a neuropeptide mainly synthesized in the brain's hypothalamic para-ventricular and supra-optic nuclei by magnocellular neurons, which is processed along the axonal projections to the posterior lobe of the pituitary [Ludwig and Leng 2006]. This neuropeptide can act on different brain areas as a neuromodulator, playing a role in several neurophysiological processes and behavior [Stoop 2012], such as feeding, anxiety, aggression, social recognition and stress response [Hashimoto et al. 2012]. Oxytocin is intricately involved in a broad array of neuropsychiatric functions and may be a common factor playing a vital role in multiple psychiatric disorders such as autism, schizophrenia, mood and anxiety disorders [Cochran et al. 2013].

Recently, the interest around oxytocin has grown, and this neuropeptide has been claimed to be a potential pharmacological target for the treatment of behavioral and cognitive symptoms in mental disorders [Chini et al. 2014; Gumley et al. 2014; Meyer-Lindenberg et al. 2011; Okamoto et al. 2016]. Central oxytocin receptors have been found in various brain regions crucial for information processing and memory, including hippocampus, striatum, amygdala, hypothalamus, 
nucleus accumbens and midbrain [Gimpl and Fahrenholz 2001]. Animal studies showed that exogenous oxytocin may have both promnestic and amnestic effects depending on timing of delivery, context, gender and dose [Bielsky and Young 2004; Chini et al. 2014]. In particular, the amnestic proprieties of oxytocin may have an important role in the context of specific reproductive conditions [Heinrichs et al. 2004].

Whereas the effects of oxytocin on memory have been thoroughly investigated in animals, research on humans is relatively limited. Studies reporting temporary verbal episodic memory impairment in women during pregnancy and post-partum [Brett and Baxendale 2001] concluded that oxytocin may have an important biological function by inhibiting acquisition of aversive experiences during labor [Heinrichs et al. 2004]. Inferences from the effects of oxytocin cannot, however, be drawn based exclusively on correlational data of endogenous physiological concentrations. More direct evidence can be obtained examining exogenous administration of oxytocin and its consequences on behavior and cognition. A recent review on studies in healthy participants administered oxytocin using a nasal spray, reported a variety of behavioral and cognitive effects in social decision making and in processing social stimuli [Macdonald and Macdonald 2010].

Despite data showing that intranasal administration of oxytocin could increase cerebrospinal fluid levels of the neuropeptide in humans [Striepens et al. 2013], the bio-availability of exogenous intranasal oxytocin in the brain remains an open question, and more work is needed to clarify this key point [Leng and Ludwig 2016; Valstad et al. 2016]. This review provides a detailed summary of the evidence of the effects of exogenous intranasal oxytocin on long-term memory in healthy human adults.

\section{METHODS}

\section{Inclusion/exclusion criteria}

We included; (1) original research studies, (2) conducted on healthy adults participants, (3) with the administration of intranasal oxytocin (single or repeated dose), which (4) provided at least one 
outcome of long-term memory and (5) were published between January 1980 and June 2016. We decided to select exclusively studies in which participants received oxytocin intranasally, since this is the most common method for oxytocin administration in human studies. Furthermore, articles published in languages other than English, as well as animal studies, reports of secondary data such as meta-analyses, reviews or letters were also excluded.

\section{Search strategy and selection of the studies}

The electronic database Medline (PubMed) was investigated for records which either contained the items "Oxytocin" in the title and "memory", "retrieval", "encoding" or "recognition" in the title or abstract. Only English-language articles and studies on human subjects were selected with a date range restricted to 1980-2016 (see Figure 1, Table 1 and Table 2). Abstracts were reviewed and all relevant original research articles were considered in detail, including a review of references from each publication to identify additional sources. References and "cited by" information of identified articles were further scanned, but no additional studies were found. Articles were then chosen by two independent reviewers based on the relevance and aim of the review, study design, quality of study and overall manuscript quality.

To focus on the impact of oxytocin on long-term memory and emotional processing, as reported in previous studies [Guastella and MacLeod 2012; Macdonald and Macdonald 2010], we identified two main categories of research: a) studies on the effect of oxytocin on long-term memory of non-emotional stimuli (e.g., non-emotional words, non-emotional objects, nonemotional neutral faces) and b), studies on the effect of oxytocin on the long-term memory of emotional stimuli (e.g., emotional words, emotional faces, emotional pictures).

\section{RESULTS}

In total, 18 articles published between 1980 and 2016 fitted the criteria and were included in the systematic review (see Figure 1). A total of 939 participants were enrolled in the selected studies 
( $87 \%$ males, $13 \%$ females).

Impact of oxytocin on long-term memory performance using non-emotional stimuli

The first studies that examined the effects of exogenous intranasal oxytocin on human longterm memory used non-emotional stimuli (i.e., words or neutral faces) (see Table 1). In the first study, two different long-term memory tests were administered during and after the assumption of oxytocin: a paired-associate word task, which required the subject to learn and recall 10 word-pairs and a neutral face picture matching task [Ferrier et al. 1980]. The authors found that the ability to recall word pairs decreased after learning, and markedly so in later recall, while no effects were detected for the picture matching task. Subsequently, Fehm-Wolfsdorf et al. [1984] demonstrated that oxytocin administered during the memory retrieval phase, but not before the encoding phase, decreased memory performance [Fehm-Wolfsdorf et al. 1988]. Bruins et al. [1992] reported that oxytocin administered before learning a word-list, reduced initial storage (i.e., correctly remembered words after the initial presentation) and the rate of storage (i.e., number of trials to recall words at least once) for verbal material in healthy young men, but no difference was detected for delayed recall.

Furthermore, Heinrichs et al. [2004] showed that in a long-term memory test, oxytocin significantly impaired recall performance compared with placebo treatment, irrespective of the meaning of the words used. Herzmann et al. [2012] directly contrasted the effects of oxytocin on the recognition of non-emotional stimuli (neutral faces and houses), showing a moderate memory impairment for both type of stimuli. In the study by Blandòn-Gitlin et al. [2014], participants received intranasal solutions of oxytocin or placebo before encoding viewing white and black neutral faces or during recognition. Only if oxytocin was administered before encoding, did it increase black neutral faces recognition, eliminating own-race bias. Finally, Bate et al. [2015] reported that participants' overall memory performance was not affected by oxytocin, but it elicited a positive response bias, i.e., participants were more likely to make false-positive recognition errors. 
In summary, studies on intranasal oxytocin effects on long-term memory for non-emotional stimuli either found a reduction of performance or no effects, with the exception of a single study that showed that performance improved, revealing a reduction of own-race memory bias during a face recognition task [Blandon-Gitlin et al. 2014].

\section{Impact of oxytocin on long-term memory performance using emotional stimuli}

More recent research has examined the impact of oxytocin on long-term memory performance using emotional stimuli such as faces with emotional expressions, emotional pictures and emotional words (see Table 2). Guastella et al. [2008] explored the effect of oxytocin administered before the encoding phase on memory of faces displaying neutral and emotional expressions (happiness or anger). During an incidental recognition test, they found oxytocin enhancement of accuracy in a recognition memory task and familiarity ratings for happy faces, but not for neutral or angry faces. In contrast, Savaskan et al. [2008] administered a single dose of oxytocin to volunteers to test post-learning phase oxytocin effects on recognition of emotional faces. Their main result was that oxytocin improved identity recognition memory and reduced the false alarm rate selectively for faces with a neutral expression or an angry expression, although this effect was absent for happy faces. Interestingly, in a study by Di Simplicio et el. [2009], it was found that the promnesic effects of oxytocin on emotional material was also found for the memory recall of words with positive valence.

Administering oxytocin during encoding, Rimmele et al. [2009] compared the effects of oxytocin on the recognition of emotional faces (i.e. social stimuli) and non-social pictures, showing an improvement of accuracy selectively for emotional faces regardless the gender and the valence. In a study by Striepens et al. [2012], participants were scanned using functional MRI (fMRI), during the encoding of aversive and neutral picture stimuli selected from the International Affective Picture System [Bradley and Lang 2007] after receiving either oxytocin or a placebo. Twenty-four hours later, on an incidental free recall task, the authors observed an increase of accuracy for 
aversive stimuli related to left insula activations and increased functional coupling between the left amygdala, left anterior insula, and left inferior frontal gyrus.

A unique study that revealed gender differences in the effect of oxytocin in memory tasks was published by Herzmann at al. [2013], in which event-related potentials (ERPs) were recorded during the learning and recognition phase, while investigating neural correlations of oxytocin's responses to memory encoding and retrieval of own and other-race emotional faces. Exogenous intranasal oxytocin increased the accuracy of familiarity judgments in both gender groups and neural correlations for this effect in ERPs related to memory encoding and retrieval were observed. In contrast, an impairment of recollection judgments was found selectively for male participants.

Weigand et al. [2013] investigated the influence of a single administration of oxytocin before the encoding phase of two doses of oxytocin before encoding and before the retrieval phase on recognition memory for neutral, positive and negative social scenes taken from the International Affective Picture System [Bradley and Lang 2007]. This study showed that only the administration of repeated assumptions of oxytocin significantly improved memory accuracy for negative social stimuli.

In Cardoso and Collaborators [2014], participants completed the Autobiographical Memory Test [Williams and Broadbent 1986] after the assumption of 24 or 48 IU of oxytocin. They found that only the 24 IU dose increased the number of specific personal memories recalled. These in turn promoted the recall of social affiliation memories that were rated more positively.

Finally, two studies applied an associative memory task that included either emotional (smiling and angry faces) or non-emotional (green and red lights) feedback [Hu et al. 2015; Hurlemann et al. 2010]. Subjects' learning performance improved when emotional rather than nonemotional feedback was provided [Hu et al. 2015; Hurlemann et al. 2010]. fMRI data showed that during the response phase, oxytocin selectively increased activity in the amygdala, hippocampus, para-hippocampal gyrus and putamen and functional connectivity between the amygdala and insula and caudate [Hu et al. 2015]. In summary, studies on intranasal oxytocin effects on long-term 
memory performance using emotional stimuli found an improvement in memory, while no studies showed any worsening of memory performance.

\section{DISCUSSION}

In this review, we have summarized the heterogeneous literature reporting intranasal oxytocin changes for long-term memory performance on healthy human subjects. The literature showed contradictory results and research designs of studies which varied widely in the methodologies used (memory and learning paradigms), therefore producing variable outcomes. The studies included here were difficult to compare due to limited oxytocin dosage range, single versus repeated administrations and stimulation protocols administered at different timings. Furthermore, a large variability of long-term episodic memory measurements was also found. These included verbal episodic memory paradigms (i.e., word-learning tasks), non-verbal memory tasks (i.e., facial or picture recognition), autobiographical memory test and associative memory task. Moreover, mainly male participants were recruited, limiting the interpretation of our findings to a malepredominant population. Interestingly, one study did show a gender difference in oxytocin response on memory abilities [Herzmann et al. 2013], in line with previous research, demonstrating that oxytocin affects social cognition aspects do differ for males and females [Domes et al. 2007a; Domes et al. 2007b; Domes et al. 2010; Macdonald and Macdonald 2010; Marsh et al. 2010].

Overall, certain studies demonstrated induced worsening of episodic memory, others showed no effect at all on memory, while other research found an improvement of memory depending on the type of task used. We found that the common distinction between memory for social and non-social stimuli was not completely satisfactory to explain the inconsistent findings of these study results. We therefore suggest a preliminary distinction between non-emotional or emotional material administered, indicating that while an oxytocin amnestic effect or no effect can be found for non-emotional stimuli (verbal, non-verbal, social or non-social) an oxytocin promnesic effect was detected systematically for emotional stimuli. 
Interestingly, in a recent study by Feifel et al. [2012] investigating the effects of three weeks of daily intranasal administrations of oxytocin in patients with schizophrenia, a beneficial effect on long-term verbal memory for non-emotional stimuli was reported. Previous studies found that the differential effects of oxytocin may depend on individual characteristics, showing that they may be more pronounced in subjects with impaired cognitive abilities, emotion disregulation and with higher score of alexithymia [Luminet et al. 2011; Quirin et al. 2014; Weigand et al. 2013]. Moreover, nasal sprays are limited in terms of controlling dosing and absorption and consequently of drug response [Chini et al. 2014; Guastella and MacLeod 2012].

Only one study, using a non-emotional facial stimuli, found any improvement of memory performance, i.e., decreased own-race bias [Blandon-Gitlin et al. 2014]. As the authors suggest, we might consider that this type of material which did not consider any facial expression, cannot be considered exactly non-emotional. This is because emotions may play an important role when the participant has to recognize other race faces, as demonstrated in the functional MRI study by Cunningham, in which amygdala activity increased more in response to black compared to white faces [Cunningham et al. 2004].

Regarding emotional stimuli, a general improvement of memory performance was detected, provoking an improvement of overall accuracy or reduction of certain types of errors or specific biases. Oxytocin did enhance memory performance for emotional face expressions, pictures and words when administered before the study phase of a long-term memory experiment. It also enhanced memory performance when administered after the study phase, suggesting that oxytocin might not enhance only encoding but also consolidation processes.

It remains unclear whether the effects of oxytocin spray could have been more specific to limited types of expressions (e.g., happy, angry) or to specific valence categories (positive vs. negative). Furthermore, it is worth noting that it is difficult to distinguish whether improvements of memory performance for emotional tasks are directly related to a memory process enhancement or are due to a primary improvement of emotion recognition abilities observed in some research. This 
may increase the salience of material and decrease the cognitive load of the learning process [Macdonald and Macdonald 2010].

To sum up, oxytocin's effects on memory can be summarized into a complex picture where facilitation or interference effects are dependent on the phase and dose of peptide administration (pre- vs. post-memory encoding) and the nature of the stimuli used (emotional vs. non-emotional). Although we have identified a link between memory performance and oxytocin in humans, conclusions that can be drawn are only tentative and need to be explored further. Oxytocin is intricately involved in a broad array of neuropsychiatric functions and it is a new pharmacological target for many trials on psychiatric disorders [Cochran et al. 2013].

We believe that in light of these considerations, clinical trials on patient populations using repeated administrations of exogenous intranasal oxytocin, should investigate all the possible amnestic and promnesic effects of the neuropeptide. Several studies have shown that long-term human memories are influenced by the emotion experienced during learning [Buchanan 2007; Phelps 2004]. Further studies need to elucidate oxytocin's effects on memory describing the effects as a function of emotional salience of the learning material and the underlying neural mechanisms. In particular, more information is needed to determine the mechanisms of the oxytocin peptide on learning and memory processes in order to assess whether oxytocin has any direct effect on the synaptic plasticity involved in long-term potentiation and/or long-term depression of synaptic transmission or on other neuromodulators.

\section{Acknowledgements}

We thank prof. Robert Coates of the Language Centre of Bocconi University of Milan for the professional English editing of the manuscript. Mauro Adenzato was supported by a MIUR grant (FIRB 2012-2017, protocol number: RBFR12F0BD_001) and by the University of Torino (Ricerca scientifica finanziata dall'Università "Cognizione sociale e attaccamento in popolazioni cliniche e non cliniche"). 


\section{References}

Bate S, Bennetts R, Parris BA, Bindemann M, Udale R, Bussunt A. 2015. Oxytocin increases bias, but not accuracy, in face recognition line-ups. Social cognitive and affective neuroscience 10(7):1010-4.

Bielsky IF, Young LJ. 2004. Oxytocin, vasopressin, and social recognition in mammals. Peptides 25(9):1565-74.

Blandon-Gitlin I, Pezdek K, Saldivar S, Steelman E. 2014. Oxytocin eliminates the own-race bias in face recognition memory. Brain research 1580:180-7.

Bradley MM, Lang PJ. 2007. The International Affective Picture System (IAPS) in the study of emotion and attention. In: Coan JA, Allen JJB, editors. Handbook of emotion elicitation and assessment. New York: Oxford University Press.

Brett M, Baxendale S. 2001. Motherhood and memory: a review. Psychoneuroendocrinology 26(4):339-62.

Bruins J, Hijman R, Van Ree JM. 1992. Effect of a single dose of des-glycinamide[Arg8] vasopressin or oxytocin on cognitive processes in young healthy subjects. Peptides 13(3):461-8.

Buchanan TW. 2007. Retrieval of emotional memories. Psychological bulletin 133(5):761-79.

Cardoso C, Orlando MA, Brown CA, Ellenbogen MA. 2014. Oxytocin and enhancement of the positive valence of social affiliation memories: an autobiographical memory study. Social neuroscience 9(2):186-95.

Chini B, Leonzino M, Braida D, Sala M. 2014. Learning about oxytocin: pharmacologic and behavioral issues. Biological psychiatry 76(5):360-6.

Cochran DM, Fallon D, Hill M, Frazier JA. 2013. The role of oxytocin in psychiatric disorders: a review of biological and therapeutic research findings. Harvard review of psychiatry 21(5):219-47.

Cunningham WA, Johnson MK, Raye CL, Chris Gatenby J, Gore JC, Banaji MR. 2004. Separable neural components in the processing of black and white faces. Psychological science 15(12):806-13.

Di Simplicio M, Massey-Chase R, Cowen PJ, Harmer CJ. 2009. Oxytocin enhances processing of positive versus negative emotional information in healthy male volunteers. Journal of psychopharmacology 23(3):241-8.

Domes G, Heinrichs M, Glascher J, Buchel C, Braus DF, Herpertz SC. 2007a. Oxytocin attenuates amygdala responses to emotional faces regardless of valence. Biological psychiatry 62(10):1187-90.

Domes G, Heinrichs M, Michel A, Berger C, Herpertz SC. 2007b. Oxytocin improves "mindreading" in humans. Biological psychiatry 61(6):731-3.

Domes G, Lischke A, Berger C, Grossmann A, Hauenstein K, Heinrichs M, Herpertz SC. 2010. Effects of intranasal oxytocin on emotional face processing in women. Psychoneuroendocrinology 35(1):83-93.

Fehm-Wolfsdorf G, Bachholz G, Born J, Voigt K, Fehm HL. 1988. Vasopressin but not oxytocin enhances cortical arousal: an integrative hypothesis on behavioral effects of neurohypophyseal hormones. Psychopharmacology 94(4):496-500.

Fehm-Wolfsdorf G, Born J, Voigt KH, Fehm HL. 1984. Human memory and neurohypophyseal hormones: opposite effects of vasopressin and oxytocin. Psychoneuroendocrinology 9(3):285-92.

Feifel D, Macdonald K, Cobb P, Minassian A. 2012. Adjunctive intranasal oxytocin improves verbal memory in people with schizophrenia. Schizophrenia research 139(1-3):207-10.

Ferrier BM, Kennett DJ, Devlin MC. 1980. Influence of oxytocin on human memory processes. Life sciences 27(24):2311-7.

Gabrieli JD. 1998. Cognitive neuroscience of human memory. Annual review of psychology 49:87- 
115.

Gimpl G, Fahrenholz F. 2001. The oxytocin receptor system: structure, function, and regulation. Physiological reviews 81(2):629-83.

Guastella AJ, MacLeod C. 2012. A critical review of the influence of oxytocin nasal spray on social cognition in humans: evidence and future directions. Hormones and behavior 61(3):410-8.

Guastella AJ, Mitchell PB, Mathews F. 2008. Oxytocin enhances the encoding of positive social memories in humans. Biological psychiatry 64(3):256-8.

Gulpinar MA, Yegen BC. 2004. The physiology of learning and memory: role of peptides and stress. Current protein \& peptide science 5(6):457-73.

Gumley A, Braehler C, Macbeth A. 2014. A meta-analysis and theoretical critique of oxytocin and psychosis: prospects for attachment and compassion in promoting recovery. The British journal of clinical psychology / the British Psychological Society 53(1):42-61.

Hashimoto H, Uezono Y, Ueta Y. 2012. Pathophysiological function of oxytocin secreted by neuropeptides: A mini review. Pathophysiology : the official journal of the International Society for Pathophysiology / ISP 19(4):283-98.

Heinrichs M, Meinlschmidt G, Wippich W, Ehlert U, Hellhammer DH. 2004. Selective amnesic effects of oxytocin on human memory. Physiology \& behavior 83(1):31-8.

Herzmann G, Bird CW, Freeman M, Curran T. 2013. Effects of oxytocin on behavioral and ERP measures of recognition memory for own-race and other-race faces in women and men. Psychoneuroendocrinology 38(10):2140-51.

Herzmann G, Young B, Bird CW, Curran T. 2012. Oxytocin can impair memory for social and nonsocial visual objects: a within-subject investigation of oxytocin's effects on human memory. Brain research 1451:65-73.

Hill SK, Reilly JL, Keefe RS, Gold JM, Bishop JR, Gershon ES, Tamminga CA, Pearlson GD, Keshavan MS, Sweeney JA. 2013. Neuropsychological impairments in schizophrenia and psychotic bipolar disorder: findings from the Bipolar-Schizophrenia Network on Intermediate Phenotypes (B-SNIP) study. The American journal of psychiatry 170(11):1275-84.

Hu J, Qi S, Becker B, Luo L, Gao S, Gong Q, Hurlemann R, Kendrick KM. 2015. Oxytocin selectively facilitates learning with social feedback and increases activity and functional connectivity in emotional memory and reward processing regions. Human brain mapping 36(6):2132-46.

Hurlemann R, Patin A, Onur OA, Cohen MX, Baumgartner T, Metzler S, Dziobek I, Gallinat J, Wagner M, Maier W and others. 2010. Oxytocin enhances amygdala-dependent, socially reinforced learning and emotional empathy in humans. The Journal of neuroscience : the official journal of the Society for Neuroscience 30(14):4999-5007.

Koen JD, Yonelinas AP. 2014. The effects of healthy aging, amnestic mild cognitive impairment, and Alzheimer's disease on recollection and familiarity: a meta-analytic review. Neuropsychology review 24(3):332-54.

Leng G, Ludwig M. 2016. Intranasal Oxytocin: Myths and Delusions. Biological psychiatry 79(3):243-50.

Lim MM, Young LJ. 2006. Neuropeptidergic regulation of affiliative behavior and social bonding in animals. Hormones and behavior 50(4):506-17.

Ludwig M, Leng G. 2006. Dendritic peptide release and peptide-dependent behaviours. Nature reviews. Neuroscience 7(2):126-36.

Luminet O, Grynberg D, Ruzette N, Mikolajczak M. 2011. Personality-dependent effects of oxytocin: greater social benefits for high alexithymia scorers. Biological psychology 87(3):401-6.

Macdonald K, Macdonald TM. 2010. The peptide that binds: a systematic review of oxytocin and its prosocial effects in humans. Harvard review of psychiatry 18(1):1-21.

Marsh AA, Yu HH, Pine DS, Blair RJ. 2010. Oxytocin improves specific recognition of positive 
facial expressions. Psychopharmacology 209(3):225-32.

Meyer-Lindenberg A, Domes G, Kirsch P, Heinrichs M. 2011. Oxytocin and vasopressin in the human brain: social neuropeptides for translational medicine. Nature reviews. Neuroscience 12(9):524-38.

Okamoto Y, Ishitobi M, Wada Y, Kosaka H. 2016. The Potential of Nasal Oxytocin Administration for Remediation of Autism Spectrum Disorders. CNS \& neurological disorders drug targets 15(5):564-77.

Petersen RC, Caracciolo B, Brayne C, Gauthier S, Jelic V, Fratiglioni L. 2014. Mild cognitive impairment: a concept in evolution. J Intern Med 275(3):214-28.

Phelps EA. 2004. Human emotion and memory: interactions of the amygdala and hippocampal complex. Current opinion in neurobiology 14(2):198-202.

Quirin M, Carter CS, Bode RC, Dusing R, Radtke EL, Tops M. 2014. The role of oxytocin and alexithymia in the therapeutic process. Frontiers in psychology 5:1074.

Rimmele U, Hediger K, Heinrichs M, Klaver P. 2009. Oxytocin makes a face in memory familiar. The Journal of neuroscience : the official journal of the Society for Neuroscience 29(1):3842.

Rock PL, Roiser JP, Riedel WJ, Blackwell AD. 2014. Cognitive impairment in depression: a systematic review and meta-analysis. Psychological medicine 44(10):2029-40.

Savaskan E, Ehrhardt R, Schulz A, Walter M, Schachinger H. 2008. Post-learning intranasal oxytocin modulates human memory for facial identity. Psychoneuroendocrinology 33(3):368-74.

Stoop R. 2012. Neuromodulation by oxytocin and vasopressin. Neuron 76(1):142-59.

Striepens N, Kendrick KM, Hanking V, Landgraf R, Wullner U, Maier W, Hurlemann R. 2013. Elevated cerebrospinal fluid and blood concentrations of oxytocin following its intranasal administration in humans. Scientific reports 3:3440.

Striepens N, Scheele D, Kendrick KM, Becker B, Schafer L, Schwalba K, Reul J, Maier W, Hurlemann R. 2012. Oxytocin facilitates protective responses to aversive social stimuli in males. Proceedings of the National Academy of Sciences of the United States of America 109(44):18144-9.

Valstad M, Alvares GA, Andreassen OA, Westlye LT, Quintana DS. 2016. The relationship between central and peripheral oxytocin concentrations: a systematic review and metaanalysis protocol. Systematic reviews 5:49.

Weigand A, Feeser M, Gartner M, Brandt E, Fan Y, Fuge P, Boker H, Bajbouj M, Grimm S. 2013. Effects of intranasal oxytocin prior to encoding and retrieval on recognition memory. Psychopharmacology 227(2):321-9.

Williams JM, Broadbent K. 1986. Autobiographical memory in suicide attempters. Journal of abnormal psychology 95(2):144-9. 